\title{
Effects of Ambient Temperature on the Performance of Turbofan Transonic Compressor by CFD Analysis and Artificial Neural Networks
}

\author{
Muhammad Umer Sohail \\ Dept. of Aerospace \& Aeronautics, \\ Institute of Space Technology, \\ Islamabad, Pakistan \\ engr.mu.sohail@gmail.com \\ Syed Hossein Raza Hamdani \\ Dept. of Aerospace \& Aeronautics, \\ Institute of Space Technology, \\ Islamabad, Pakistan \\ hraza@hotmail.com
}

\author{
Muhammad Hassan \\ Dept. of Electrical Engineering, \\ University of Paderborn, \\ Paderborn, Germany \\ m.hassan057@gmail.com \\ Khalid Parvez \\ Dept. of Aerospace \& Aeronautics, \\ Institute of Space Technology, \\ Islamabad, Pakistan \\ khalidparvez2009@hotmail.com
}

\begin{abstract}
The unfavorable effects of non-uniform temperature inlet flow on gas turbine engine operations have always been a hindrance on the performance of turbo-fan engines. The propulsive efficiency is a function of the overall efficiency of turbofan engine which itself is dependent on other ambient parameters. Variation of inlet compressor temperature due to increase or decrease of aircraft altitude, air density, relative humidity, and geographical climate conditions affects the compressor performance. This research focuses on the turbofan transonic compressor performance due to ambient temperature distortion. A novel predictive approach based on neural network model has been implemented to predict the compressor performance and behavior at different ambient temperature conditions. The model produces substantially accurate results when compared to the results of CFD analysis. Computational results from CFD analysis show that engine thrust decreases at higher altitude, lower density and lower pressure regions.
\end{abstract}

Keywords-transonic compressor; temperature distortion; CFD; artificial neural networks; deep learning

\section{INTRODUCTION}

Among the air breathing jet engines, turbofans are widely used in civil and military aircraft propulsion systems. In recent researches on the design and development of the transonic compressor rotor turbofan propulsive system, a continuous problem has been encountered in non-uniform inlet flow that triggers the performance of compression system on its stability. Gas turbines usually operate close to their limiting temperature where ambient temperature is very high. High ambient temperature deteriorates engine performance and stability. The primary concern of distorted inlet temperature is the ingestion of hot gasses from the environment. Compressor performance and stability deteriorated by inlet airfoil distortion, velocity distortion, reduced airflow, temperature and pressure circumferential distribution, while high ambient temperature, high absolute humidity, and low air density not only decrease compressor stall margin, but also increase fuel volatility [1-4]. If an engine is supercharged or turbocharged, then the variation of the air density does not affect the power output of the engine, until it reaches a certain altitude where even a turbocharged engine cannot compensate anymore for the loss of air density. The ideal ambient temperature at sea level does not always remain constant, as ambient temperature varies not only with altitude, but also with latitude. At high ambient temperature, air density decreases, reducing the air-fuel mixture for combustion and resulting in decrease of lift, thrust, and aerodynamic drag. High humidity causes engine icing which reduces compressor surge discharge pressure and flow capacity while it increases vibration and power consumption. In high bypass turbofan engines, super-cooled water droplets cause icing on engine inlet duct, the leading edge of fan blades, and IGV's. Aircrafts always require higher takeoff speed at low air density to compensate for the lift force [5-8].

In the past, machine learning has gained importance in the field of turbo machinery. Various machine learning techniques have been used in this field to enhance and predict behavior and performance at different operating conditions. Deep learning has recently been used to predict the pressure distribution at turbine blade and three different deep neural networks were built and trained to predict the pressure distributions of turbine airfoils in [9]. In [10], authors studied the geometric optimization of an axial compressor blade by genetic algorithm, whereas, RANS analysis and Pareto-optimal design has been solved to obtain total pressure and adiabatic efficiency as objective functions and flow field inside the compressor annulus. Brain emotional learning based intelligent 
controller has been studied to produce all-inclusive centrifugal compressor performance map incorporating speed lines that are not available in the provided curves. This technique is used to evaluate the constant and variable shaft speed, effects of load lines, and throttle valves for dynamic response of centrifugal compressor. To control compressor surge, a neuro fuzzy controlling scheme has been used in [11]. Recently, a gene expression programming to train CFD based machine learning neural network was developed for RANS turbulence model of high-pressure turbine blades. This explicit framework model could deduce wake prediction of the CFD based model [12].

To the best of our knowledge there is no published work that relates the transonic axial compressor and artificial neural networks (ANNs) to predict the performance and behavior of the compressor at variable ambient temperature. This paper presents a study which uses ANNs to predict pressure ratio, temperature ratio, efficiency, and mass flow rate distribution of three-dimensional compressor rotors, as part of a larger project called "System Identification Development for Analysis of Transonic Axial Compressor Rotor 67".

\section{METHODS}

Research is conducted on the first stage rotor of the two stage turbofan transonic compressor. Due to its high mass flow rate at designed pressure ratio, it is widely researched in aerospace industry. Initially, computational analysis is conducted on an ISA model which is considered at sea level at $288.15 \mathrm{~K}$ ambient temperature and $1 \mathrm{~atm}$ pressure, as it is used for engine performance comparison with extreme ambient temperature conditions. Further calculations are done at completely dry air where there is no considerable humidity change. Due to considerable diversity of ambient temperatures at high or low altitude and seasonal effects, very cold, moderate and extreme hot ambient temperature have also been considered. Inlet temperature of the compressor varied as per the change in ambient temperature; therefore, it changes the air density according to the Sutherland constant. The adopted pressure changes were kept the same as for the standard atmosphere. For the ANN model, the dataset is comprised of the computational parameters required in CFD analysis. Temperature values $(223 \mathrm{~K}-330 \mathrm{~K})$ and ambient pressure values (1.0atm-1.23atm) constitute the input features of the ANN model. Table I shows the dataset division and the number of samples for each category.

TABLE I. DATASET DIVISION

\begin{tabular}{|c|c|c|}
\hline Data set & Dataset division & Total samples \\
\hline Training data & $90 \%$ & 360 \\
\hline Test data & $10 \%$ & 42 \\
\hline
\end{tabular}

The aim is to perform prediction based on the input feature set. Thus, prediction analysis provokes the network to use mean square error (MSE) as an evaluation metric [13]. For the training of the ANN, the iterative minimization of cost function is utilized. During the training phase, ADAM optimizer has been used to update the weights and biases of the network. The weights are initialized from a random normal distribution. The initial learning rate value is set to 0.01 and $\beta_{1}$ and $\beta_{2}$ values are set to 0.9 and 0.999 respectively [14]. For generalization purposes, the training data are randomly shuffled and standardized to zero mean and unit variance. The network has been trained for 100 epochs. Keras has been opted as a framework for implementing the neural network architecture. The chosen library is a popular framework for rapid prototyping and developing high-level modularity of ANNs. Table II shows the network architecture and hyper parameters.

TABLE II. NETWORK ARCHITECTURE AND HYPER PARAMETERS

\begin{tabular}{|c|c|}
\hline Network design & Model \\
\hline Hidden Layers & 2 \\
\hline Learning rate & 0.01 \\
\hline Batch size & 16 \\
\hline Hidden layers non-linearity & ReLU \\
\hline Drop out & 0 \\
\hline
\end{tabular}

\section{COMPUTATIONAL SETUP}

Transonic compressor NASA rotor 67 is composed of 22 blades and at leading and trailing edges. It has $25.7 \mathrm{~cm}$ and $24.25 \mathrm{~cm}$ tip radii respectively as shown in Figure 1. Three dimensional steady compressible Navier-Stoke equation is solved using the $K-\varepsilon$ turbulence model. At the boundaries of rotor computational domain, three coefficients for ideal gas standard boundary conditions are applied. Furthermore, no slip conditions were used on walls whereas periodic conditions were applied at the periodic surfaces. For grid independence study, 3D mesh at coarse, medium, fine, and super fine state is generated. For grid analysis, computations are carried out to 0.4 million to 1.6 million mesh nodes. Figure 2 shows the fine hexahedral-grid mesh with 0.96 million mesh elements which has decent justification between computational and NASA experimental results.

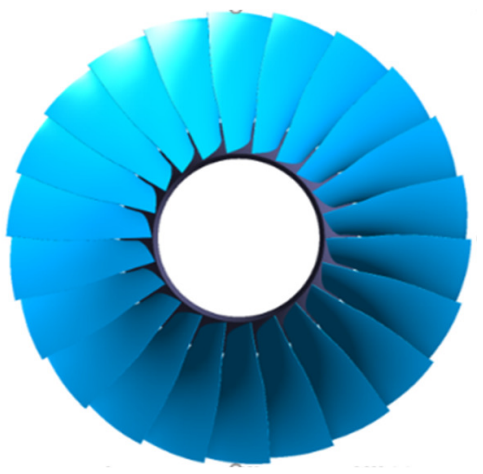

Fig. 1. Transonic rotor geometry

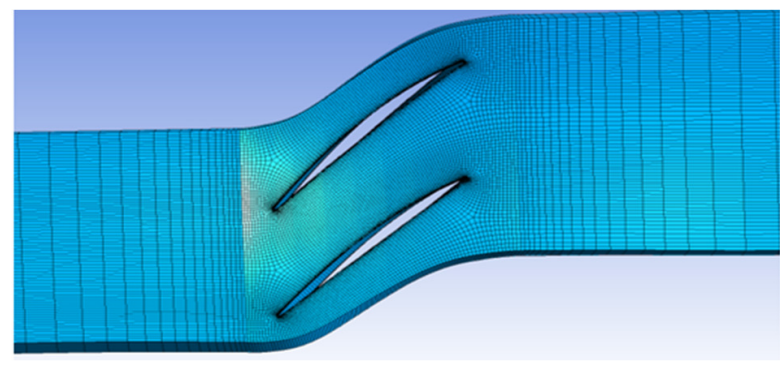

Fig. 2. Fine mesh of rotor 67 


\section{RETSULTS AND DISCUSSION}

\section{A. Computational Validation}

Computations were carried out from choking to near stall conditions by gradually raising the outlet average static pressure to obtain compressor rotor characteristic graph at sea level. The near stall point is predicted at the last stable condition of the rotor 67 . Figure 3 shows the characteristic map of compressor at designed $16043 \mathrm{rpm}$ and at tip clearance $1.016 \mathrm{~mm}$ that illustrates good validation between computational and NASA experimental results at sea level. Computational results show $33.14 \mathrm{~kg} / \mathrm{s}$ mass flow rate at the designed condition of $0.3 \%$ difference from NASA experimental results, which is fairly encouraging.

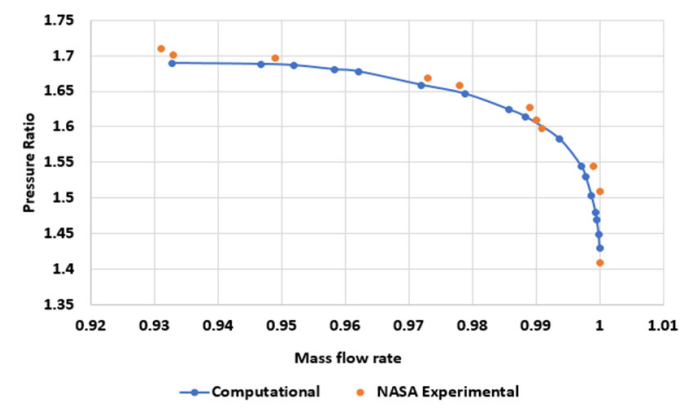

Fig. 3. Characteristic map at sea level

\section{B. Results at Sea Level Condition}

Figure 4 shows static temperature contours, Mach number contours, pressure and density contours at suction and pressure side of the blade at designed tip clearance of $1.016 \mathrm{~mm}$ and $16043 \mathrm{RPM}$ at sea level condition where $T_{\text {in }}$ is $288.15 \mathrm{~K}$, near stall point. The following conclusions have been drawn:

- Temperature gradually starts increasing at the mid of the span to tip region. Temperature contours show almost the same behavior at the suction side of the blade where there is a high temperature region above $90 \%$ span of the rotor blade.

- Mach number remains subsonic near the hub of the blade. Flow becomes transonic at nearly $50 \%$ of the blade span, and at $90 \%$ of the blade span it becomes nearly supersonic at the leading edge (LE) of the suction side. The flow behavior at pressure side is almost similar to the suction side of the blade.

- Pressure gradually rises from hub to shroud as there is a low pressure region near the hub of the blade and a very high-pressure region that is above $80 \%$ of the span on the pressure side of the blade.

- There is a low density region at the LE of the suction side of the blade and a high density region at the LE of the pressure side of the blade.

\section{Results at Very Low Ambient Temperature}

Figures 5-7 show density, pressure and Mach number contours at suction and pressure side of the blade at near stall point side during very low ambient temperatures.
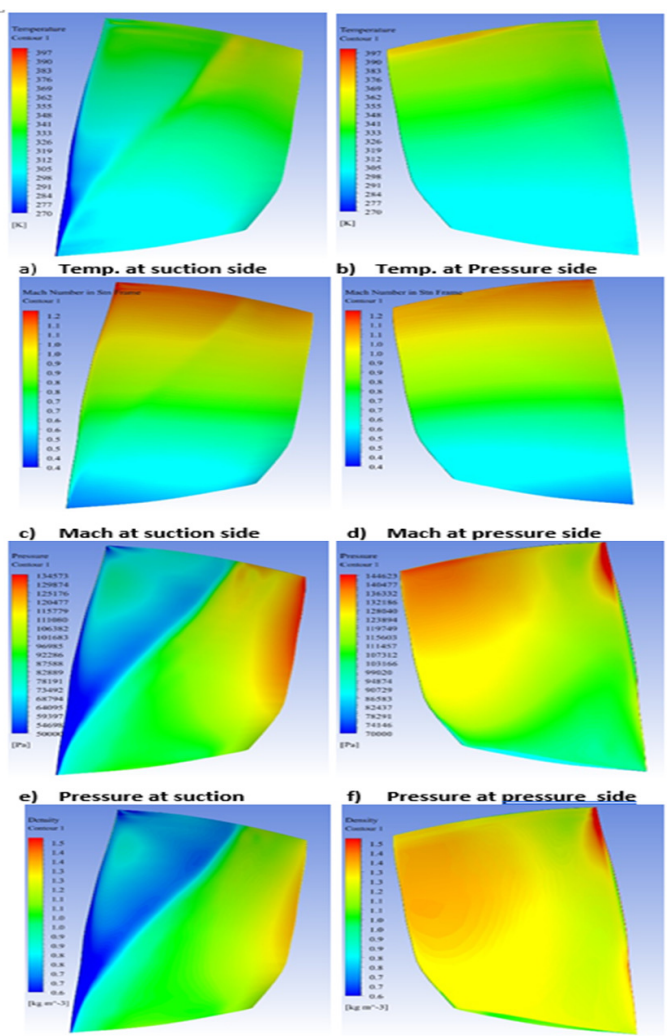

Fig. 4. Blade suction and pressure side flow contours at sea level
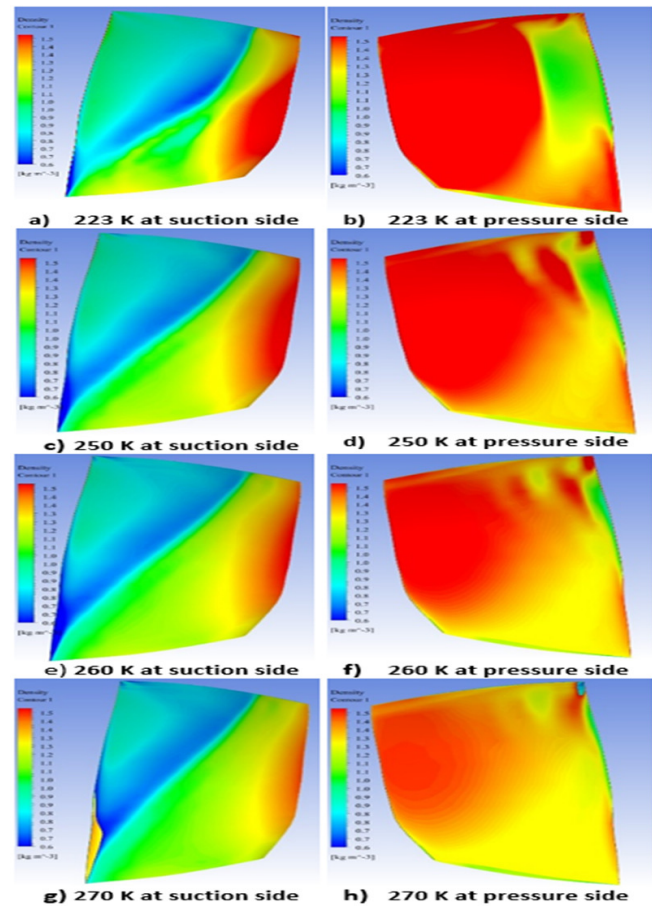

Fig. 5. Density contours at very low ambient temperatures 


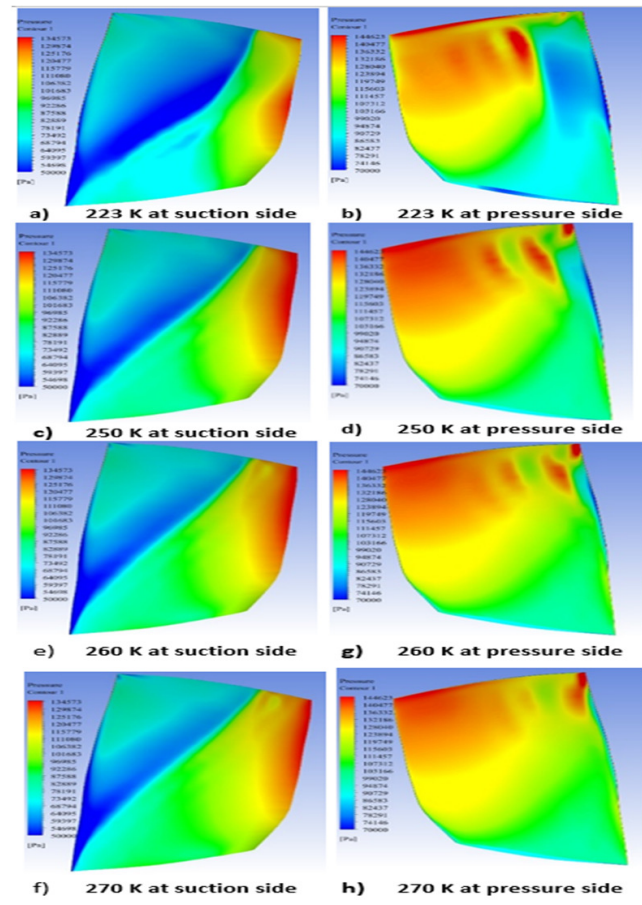

Fig. 6. Very low ampient temperature pressure contours

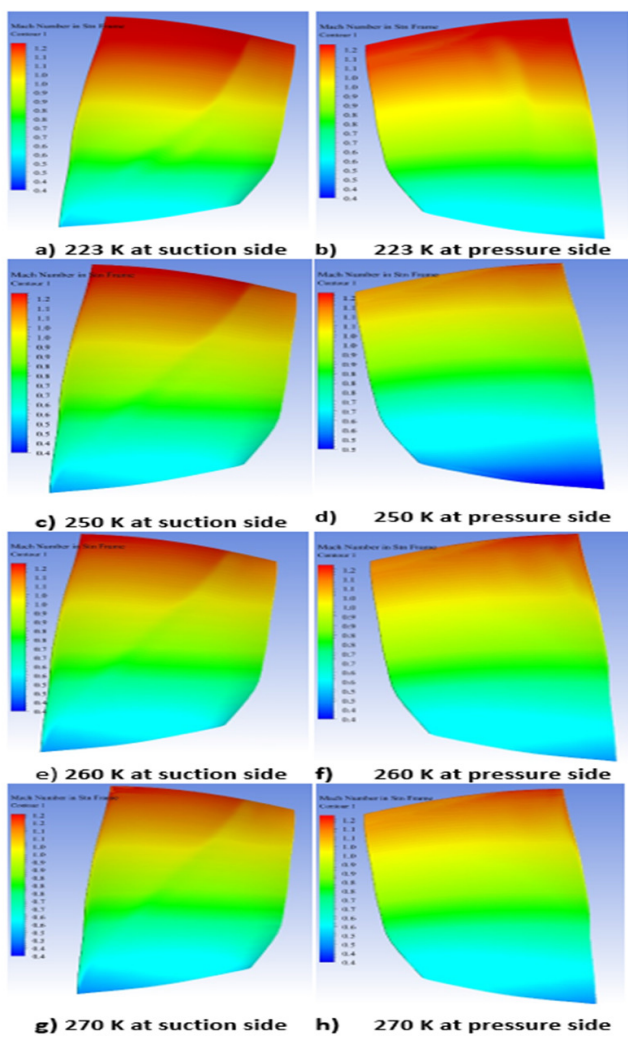

Fig. 7. Very low ampient temperature Mach contours

The following computational trends have been observed:
- Air becomes very dense at extremely low ambient temperatures. Denser air is quite dominant at the blade pressure side.

- There are high pressure contours at very low ambient temperatures. The pressure region near the hub is almost similar to the sea level, but it is higher at lower temperatures near the tip region.

- There are high Mach numbers at the blade suction and pressure side near the tip region at low ambient temperatures. Furthermore, stronger oblique shock is observed near the tip region which starts decreasing as temperature increases.

\section{Results at Moderate Ambient Temperature}

In comparison with compressor rotor behaviour at sea level, the following computational trends have been seen at blade suction and pressure side of 8-moderate ambient temperatures. Figures 8-10 show density, pressure and Mach number contours at moderate ambient temperatures.
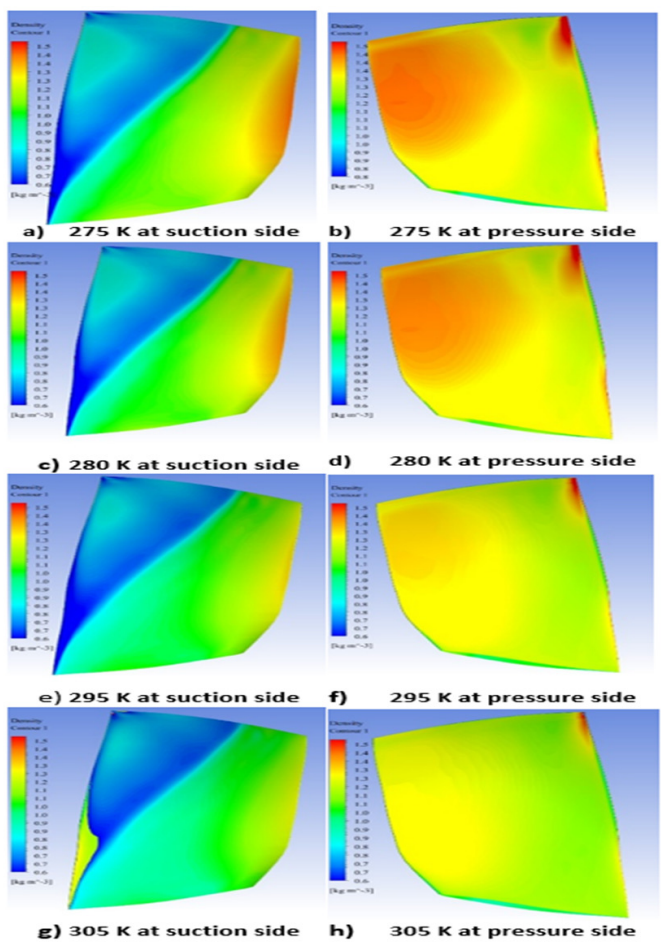

- Air density starts decreasing as temperature increases. The density at LE of the blade pressure side is slightly higher than the pressure of the suction side.

- Pressure region has similar behavior at sea level near the hub to $50 \%$ span of the blade. It is slightly greater at ambient temperature lesser than the sea level one and it decreases as temperature increases.

- There are weak oblique shock waves near the LE of the blade suction side as temperature increases. 

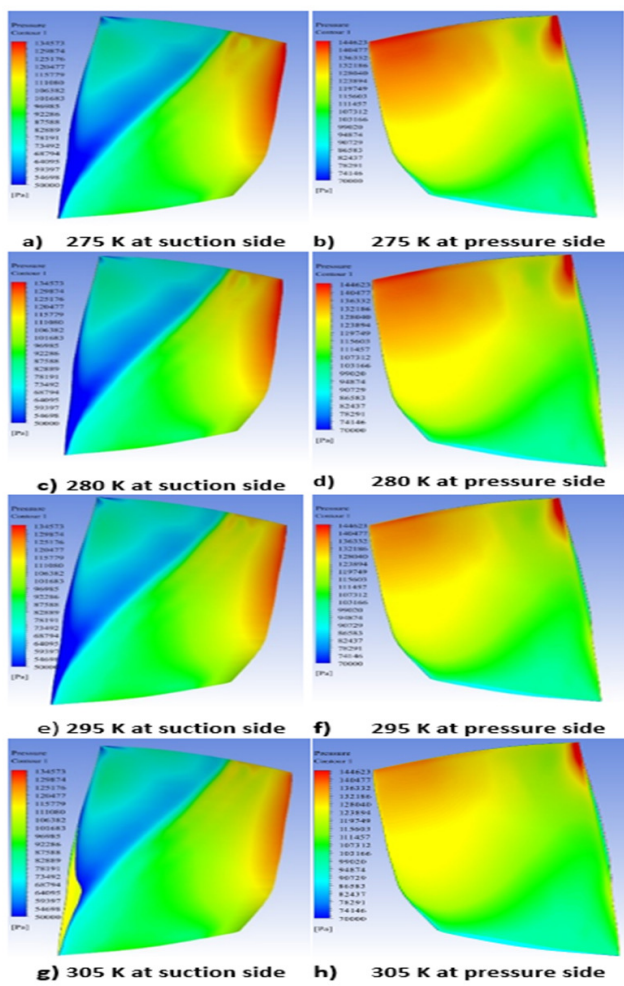

Fig. 9. Pressure contours at moderate ampient temperature
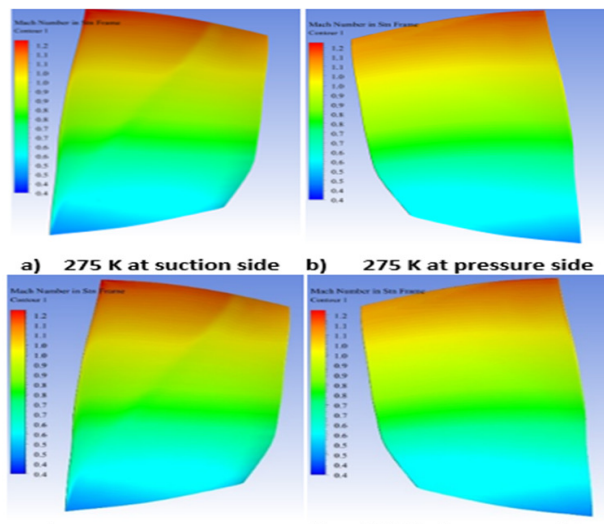

c) $280 \mathrm{~K}$ at suction side
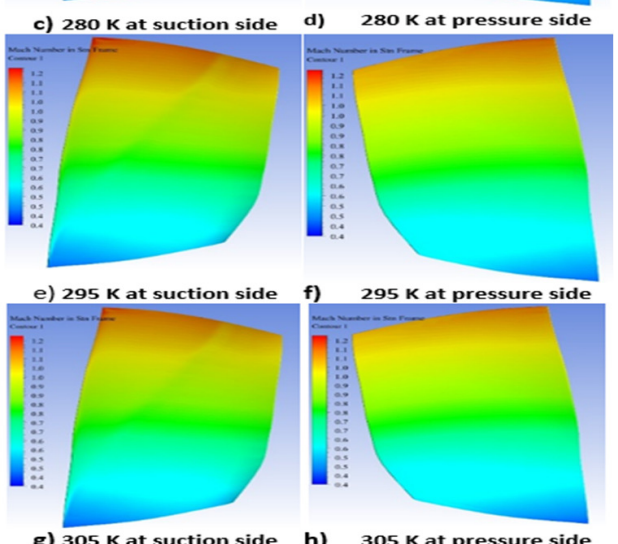

g) $305 \mathrm{~K}$ at suction side h) $305 \mathrm{~K}$ at pressure side

Fig. 10. Mach contours at moderate ampient temperature

\section{E. Results at Very High Ambient Temperature}

In comparison with compressor rotor behavior at sea level, the following computational trends have been seen at the blade suction and the pressure side of high ambient temperatures (Figures 11-13).
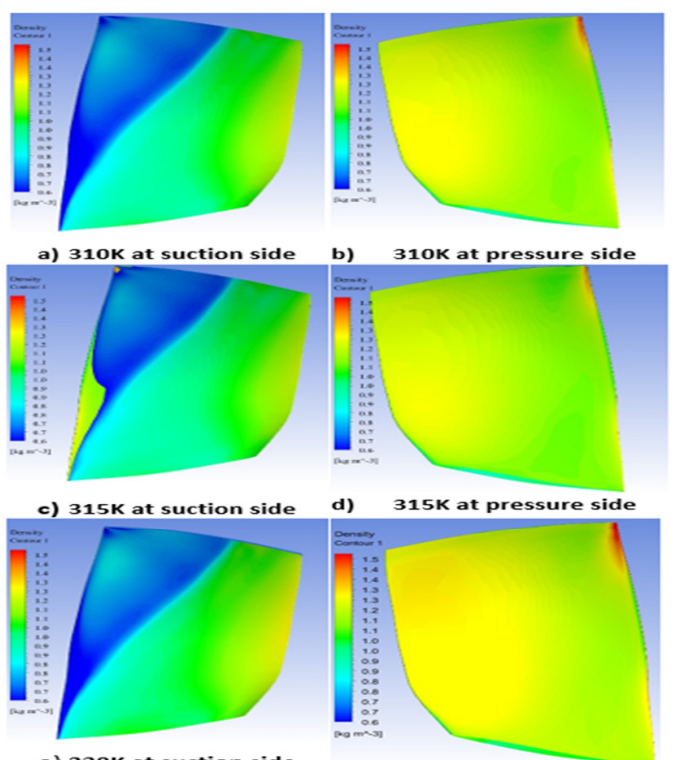

e) $320 \mathrm{~K}$ at suction side

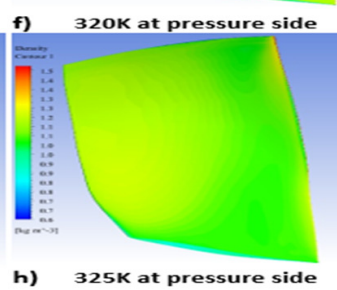

Fig. 11. Density contours at extreme temperature

- There are weak density regions at the blade suction side which further weaken as temperature increases.

- At higher ambient temperatures the air pressure decreases as air density also decreases which deteriorates the compressor performance and stability.

- Results show marginal oblique shock waves near the LE of blade suction side as temperature increases.

- There are weak density regions at the blade suction side which further weaken as temperature increases.

- At higher ambient temperatures the air pressure decreases as air density also decreases which deteriorates the compressor performance and stability.

- Results show marginal oblique shock waves near the LE of blade suction side as temperature increases.

\section{F. Effect of Ambient Temperature on Blade Loading}

Quantitative comparison of pressure variation on a single blade at $80 \%$ of its total span near stall condition at extremely low, moderate, and high ambient temperatures is shown in Figure 14. 

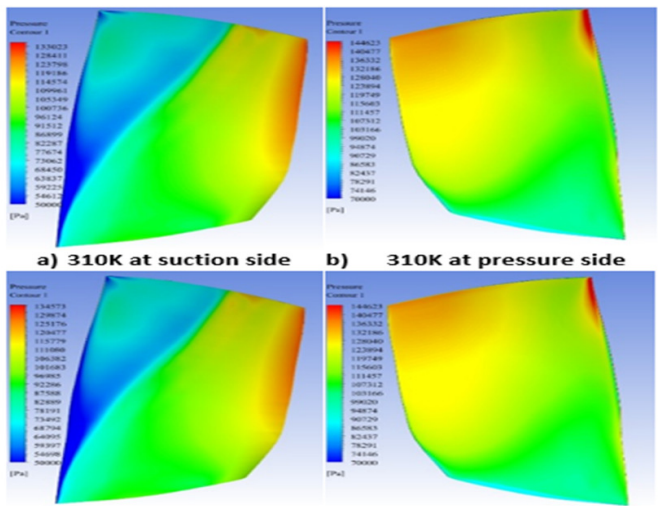

c) $315 \mathrm{~K}$ at suction side
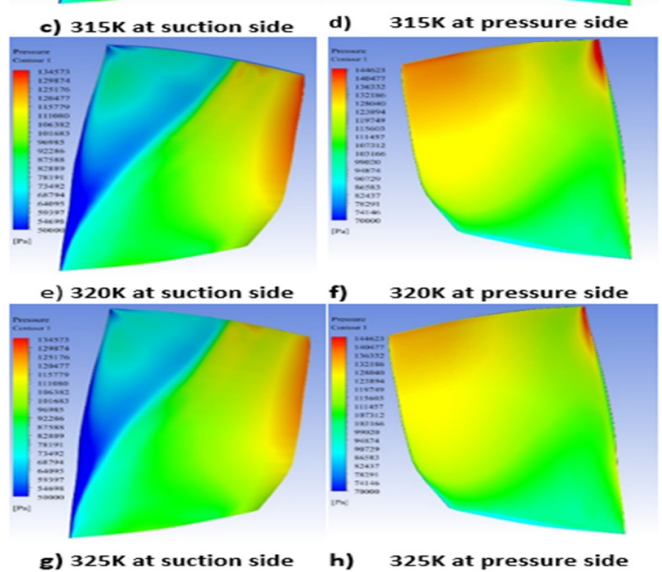

Fig. 12. Pressure contours at extreme temperature
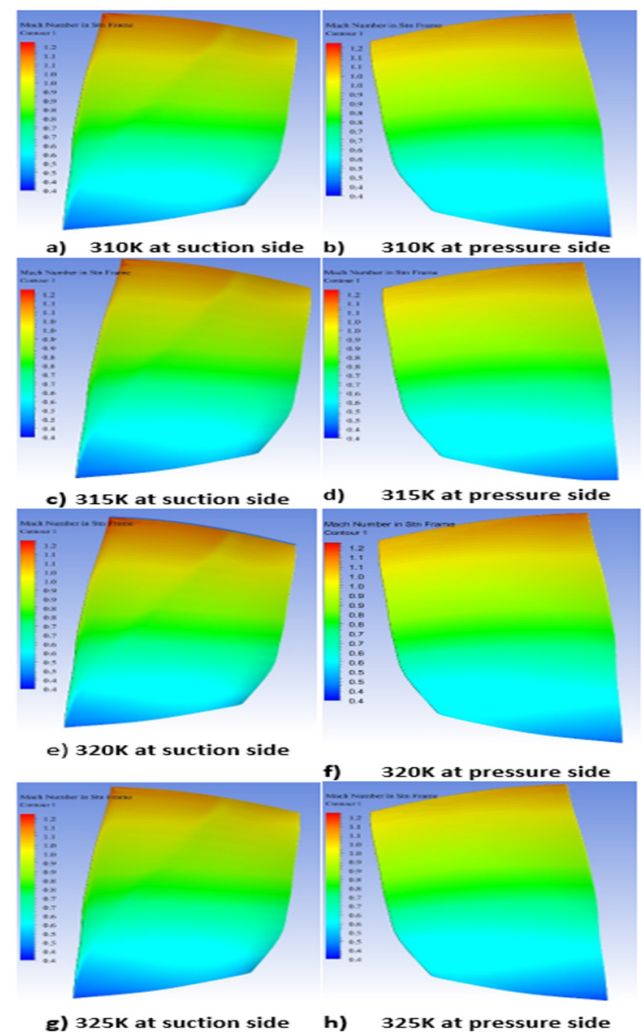

Fig. 13. Mach contours at extreme temperature

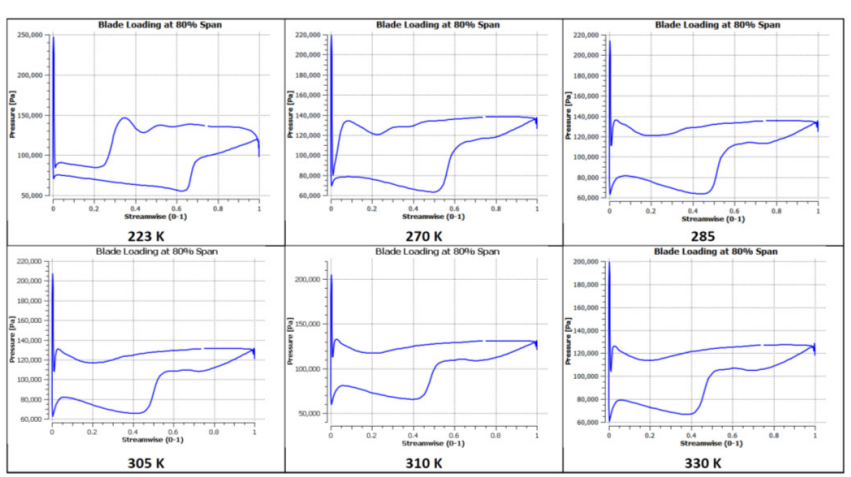

Fig. 14. Blade loading at $80 \%$ span

Blade loading is an important factor that occurs due to decrease in static pressure of incoming fluid at pressure and suction side of blade. The upper portion in the blade loading shows the pressure side and the lower portion represents the suction side of the blade. Results show almost similar trends of temperatures at the suction surface of blade loading. At very low ambient temperature, the loading at pressure surface is greater, decreasing by increasing ambient temperature. Figure 15 shows the Mach number contours on a single blade at extremely low, moderate, and high ambient temperatures. Results show high Mach number along the normalized span wise at very low ambient temperature. Mach number slightly decreases at higher ambient temperature.

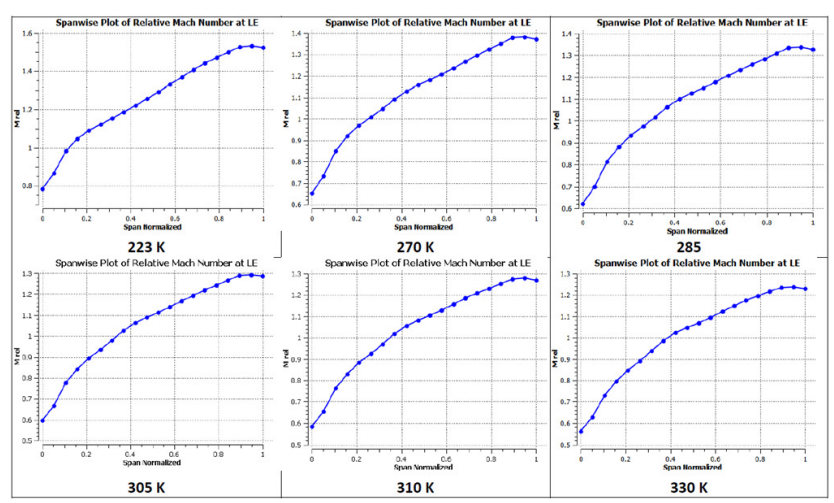

Fig. 15. Relative Mach number span wise at LE

Figure 16 shows the velocity vectors at $80 \%$ span of the rotor blade near stall condition at extremely low, moderate, and high ambient temperatures. The Figure shows high visible oblique shock waves at LE of suction side of the blade, whereas, oblique shock travels from the suction side of the first blade to the LE of the adjacent blade which results in flow blockage. Results show that at very low ambient temperature, the oblique shock is quite stronger at the suction side of the blade LE, and it becomes weaker at higher ambient temperatures. Figure 17 shows the ambient temperature vs altitude in troposphere region. Figure 18 shows the indirect relation of static pressure with altitude. It reveals that static pressure decreases at higher altitude. Figure 19 shows the variation of density with altitude. 


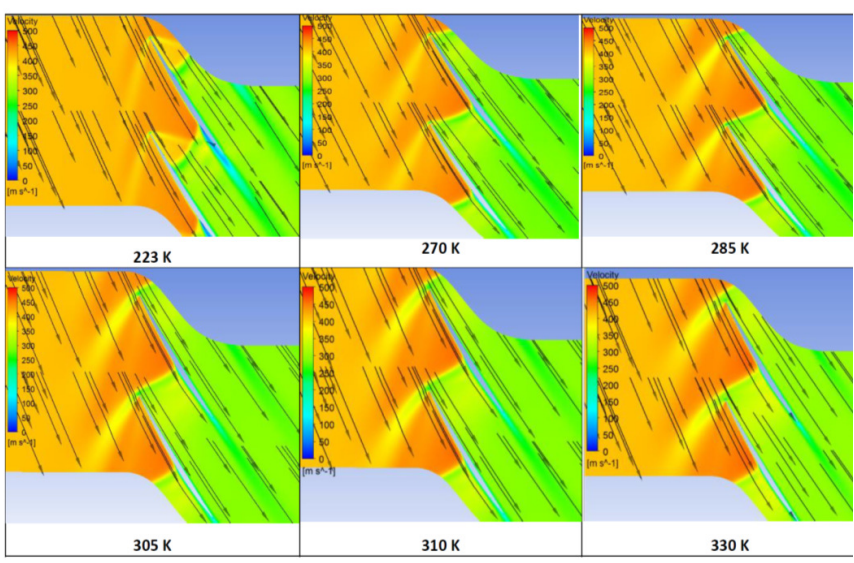

Fig. 16. Velocity vector at $80 \%$ span

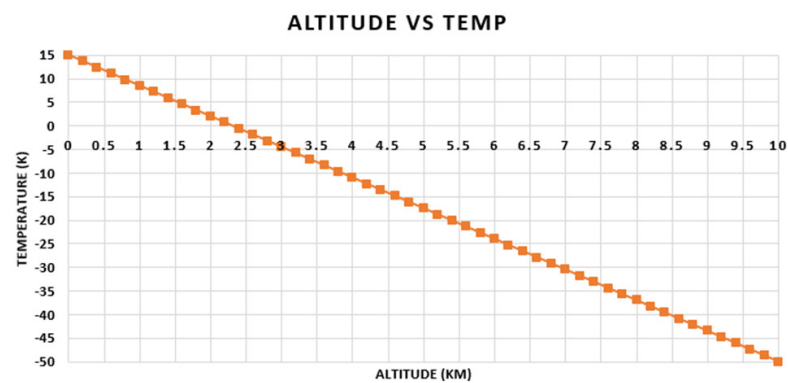

Fig. 17. Altitutude vs ambient temperature

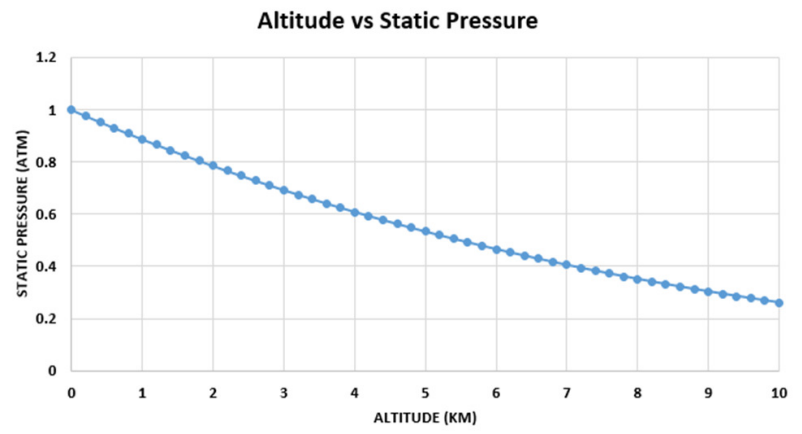

Fig. 18. Altitutude vs static temperature

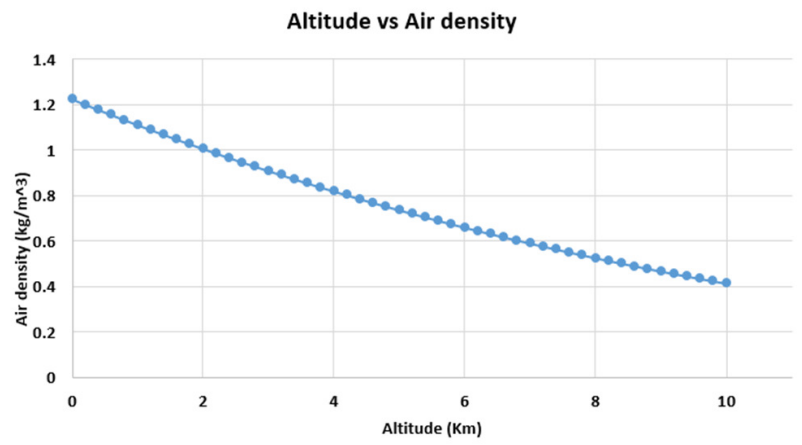

Fig. 19. Altitude vs air density

\section{G. Compressor Characteristic Map}

Figures 20-21 show the characteristic maps of compressor mass flow rate with pressure ratio, and temperature ratio respectively, to evaluate the performance of transonic compressor at different ambient temperatures, i.e. above and below sea level, whereas, Figure 22 shows the decrease of maximum mass flow rate as the ambient temperature increases. Results show that maximum mass flow rate, pressure ratio, and temperature ratio decrease at higher ambient temperature, which deteriorates the compressor stability range and performance in troposphere region ranges from sea level to above $10 \mathrm{~km}$ at steady RPM.

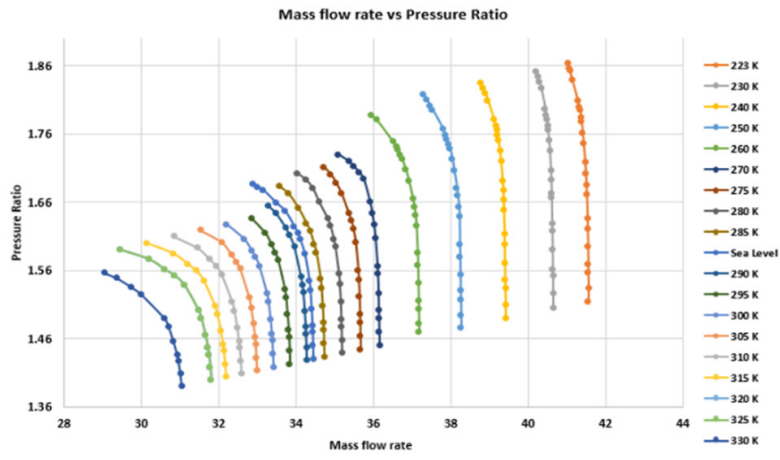

Fig. 20. Characteristic map (mass flow rate vs pressure ratio)

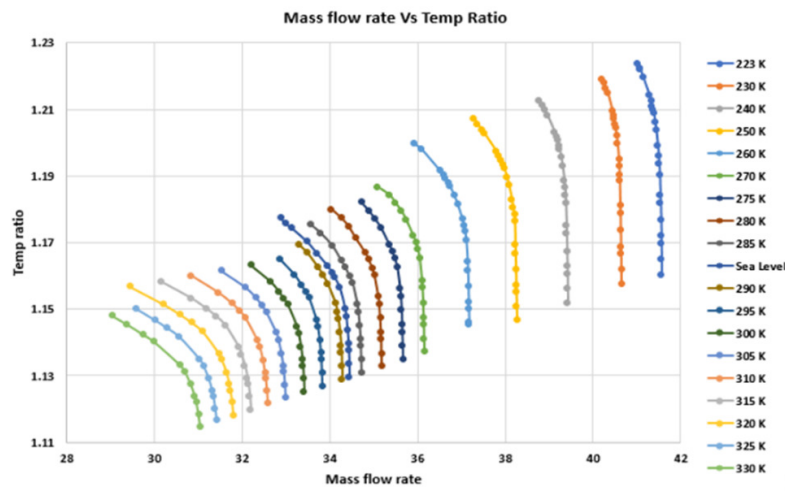

Fig. 21. Characteristic map (mass flow rate vs temperature ratio)

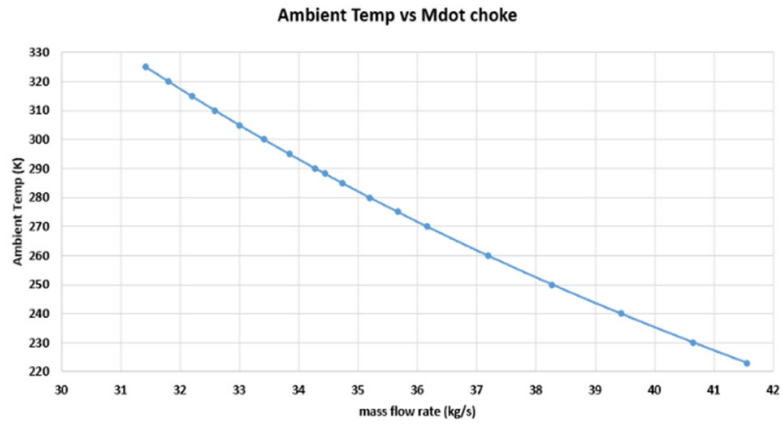

Fig. 22. Mass flow rate vs temperature 


\section{H. Neural Network Loss and Data Visualization}

Table III shows the mean square error (MSE) value during train and test phase. The values signify the generalization of the trained model on the test dataset, as the value is quite close to the train data MSE value. Figure 23 shows the MSE loss function of the model during train and test phase. The result shows that the loss function settles down during an early phase of training.

TABLE III. MSE VALUE AT TRAIN TEST PHASE

\begin{tabular}{|c|c|}
\hline Dataset division & MSE value \\
\hline Train & 0.045 \\
\hline Test & 0.052 \\
\hline
\end{tabular}

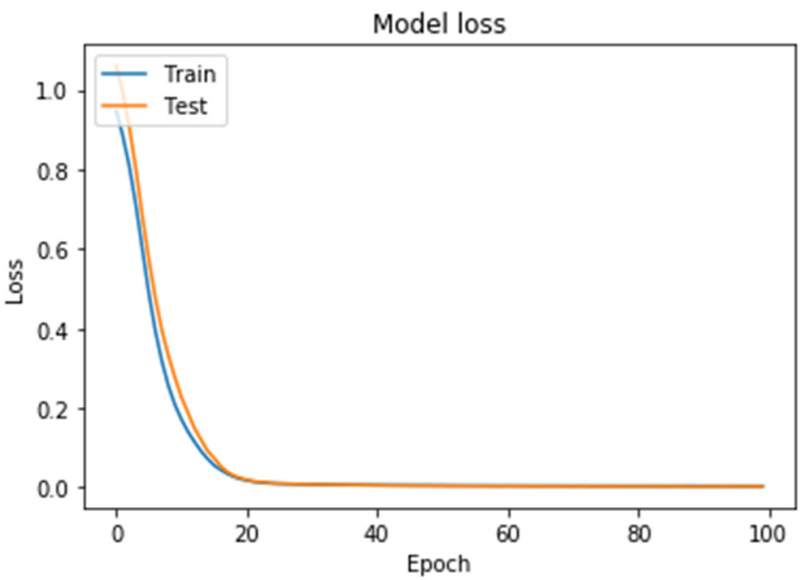

Fig. 23. Loss function during train and test phase

Figures 24-27 show the test data unity plots of mass flow rate, pressure ratio, temperature ratio and efficiency. The $\mathrm{x}$-axis corresponds to target values of the test dataset while the y-axis shows the predicted values that have been obtained when the trained model was tested with the test data set. The unity plot elaborates the performance of the network when the trained model is passed through an unseen test dataset. The difference between the predicted values (test dataset values) obtained from the trained model and the target values should be as small as possible. This results in the set of points that should ideally lie on the unity line.

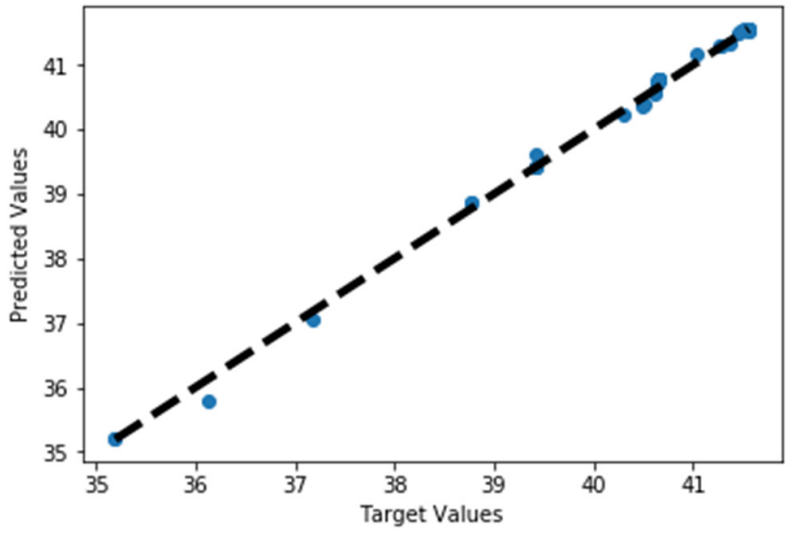

Fig. 24. Unity plot of test data mass flow rate values

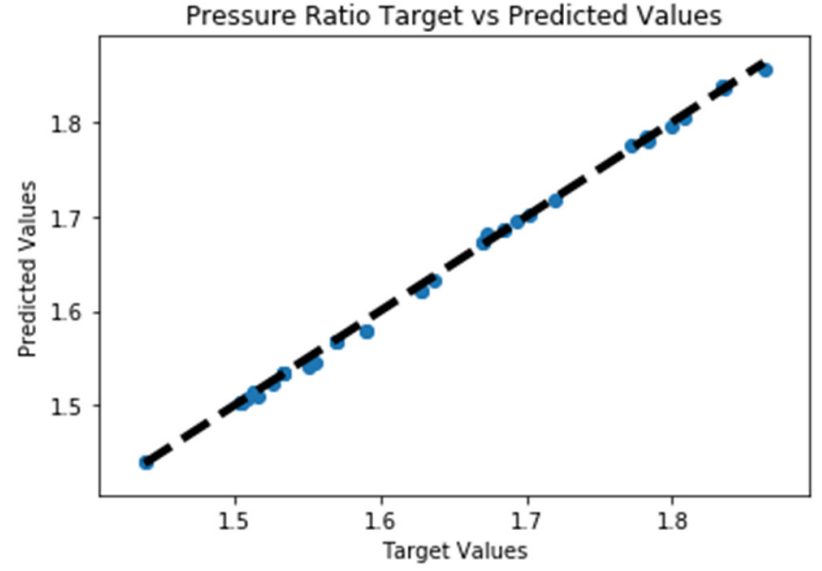

Fig. 25. Unity plot of test data pressure ratio values

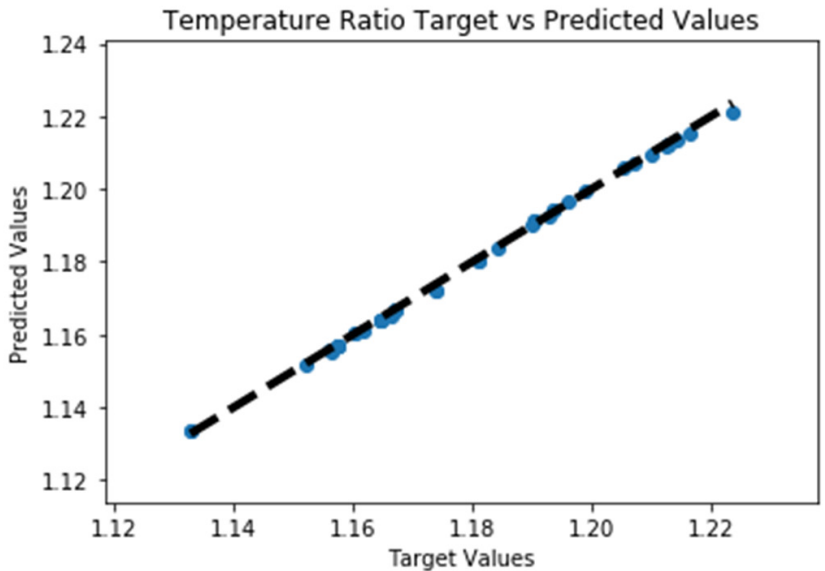

Fig. 26. Unity plot of test data temperature ratio values

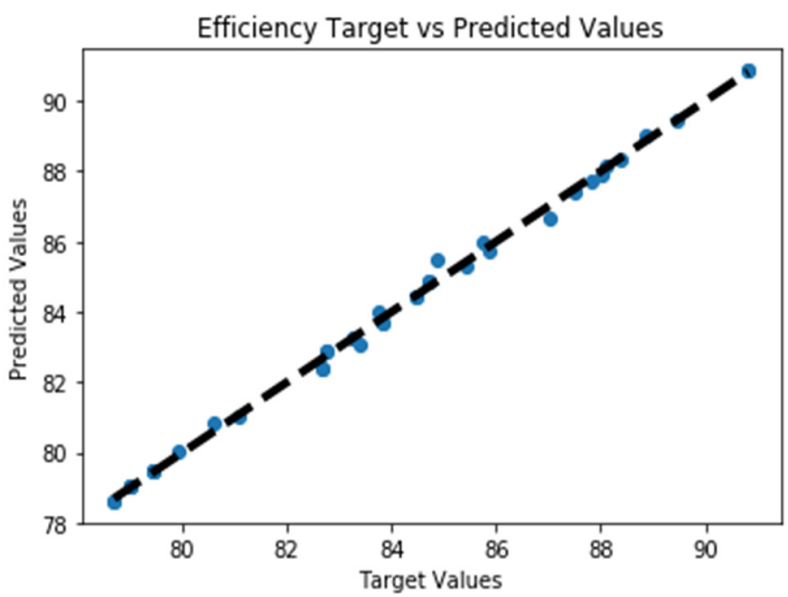

Fig. 27. Unity plot of test data efficiency values

\section{CONCLUSION}

The effects of total temperature inlet distortions on the performance of an axial compressor rotor blade row were studied using computational fluid dynamic and deep learning techniques. In transonic compressor, besides high altitude, 
humidity, low air pressure and density, ambient temperature also play a vital role in its performance. Under the influence of extremely high inlet compressor temperature, the engine may shutdown due to compressor instability. The results show that compressor rotor mass flow rate, efficiency, pressure ratio, and temperature ratio have been significantly reduced when the ambient temperature increased excessively. Very low ambient temperature and denser air produce higher mass flow rate, pressure ratio, and greater compressor efficiency. On the other hand, the results obtained from the ANN show that despite of using the conventional method of predicting parameters through CFD analysis, the use of ANNs is a promising approach to predict the parameters of compressor rotor blade. The results visualized through unity plots are a clear indication that given any set of temperature and pressure values, the trained model can accurately generate predictions of mass flow rate, temperature ratio, pressure ratio, and efficiency in less computational time as compared to simulating the models through CFD analysis.

\section{REFERENCES}

[1] H. Zhou, F. Yu, K. Yang, "Study on Design Compliences of Civil Turbofan Engine with the requirements defined in FAR 33.65", Procedia Engineering, Vol. 80, pp. 183-192, 2014

[2] W. Balicki, P. Glowacki, S. Szczecinski, R. Chachurski, J. Szczecinsk, "Effect of the Atmosphere on the Performances of Aviation Turbine Engine”, Acta Mechanica et Automatica, Vol. 8, No. 2, pp. 70-73, 2014

[3] T. K. Ibrahim, M. M. Rahman, O. M. Ali, F. Basrawi, R. Mamat, "Optimum Performance Enhancing Strategies of the Gas Turbine Based on the Effective Temperatures", MATEC Web of Conferences, Vol. 38, Article ID 01002, 2016

[4] N. R. Smith, R. A. Berdanier, J. C. Fabian, N. L. Key, "Reconciling Compressor Performance Differences for Varying Ambient Inlet Conditions", Journal of Engineering for Gas Turbines \& Power, Vol. 137, No. 12, Article ID 122603, 2015

[5] S. L. Dixon, C. A. Hall, Fluid Mechanics and Thermodynamics of Turbomachinery, Elsevier, 2005

[6] R. Stasyshan, N. Breedlove, "How Inlet conditions impact on Centrifugal Air compressor", available at: www.airbestpractices.com/ technology/air-compressors/how-inlet-conditions-impact-centrifugal-aircompressor-performance

[7] T. K. Ibrahim, M. M. Rahman, M. K. Mohammed, F. Basrawi, "Statistical analysis and optimum performance of the gas turbine power plant", International Journal of Automotive and Mechanical Engineering, Vol. 13, No. 1, pp. 3215-3225, 2016

[8] A. Razak, "Simulating the effect of change in ambient pressure on engine performance", in: Industrial Gas Turbines, Woodhead Publishing, pp. 293-322, 2007

[9] Z. Liu, X. Liu, K. Wang, Z. Liang, J. A. F. O. Correia, A. M. P. De Jesus, "GA-BP Neural Network-Based Strain Prediction in Full-Scale Static Testing of Wind Turbine Blades", Energies, Vol. 12, No. 6, Article ID 1026, 2019

[10] A. Samad, K. Y. Kim, "Shape optimization of an axial compressor blade by multi-objective genetic algorithem", Proceedings of the Institution of Mechanical Engineers Part A: Journal of Power and Energy, Vol. 222, No. 5, pp. 599-611, 2008

[11] A. Jokar, R. Zomorodian, M. G. Ghofrani, P. Khodaparast, "Active control of surge in centrifugal compressors using a brain emotional learning-based intelligent controller", Proceesing of the Institution of Mechanical Engineers Part C: Journal of Mechanical Engineering Science, Vol. 230, No. 16, pp. 2828-2839, 2016

[12] Y. Zhao, H. D. Akolekar, J. Weatheritt, V. Michelassi, R. D. Sandberg, "Turbulence Model Development using CFD-Driven Machine Learning", available at: https://arxiv.org/abs/1902.09075, 2019
[13] S. A. Gandhi, C. V. Kulkarni, "Why SSIM? - A Full Reference Image Quality Assessment", International Journal of Electronics and Communication Engineering, Vol. 2, No. 2, pp. 135-142, 2013

[14] I. Goodfellow, Y. Bengio, A. Courville, Deep Learning, MIT Press, 2016 